\title{
BMJ Open Utilizing a 3S (strategies, source and setting) approach to understand the patient's preferences when addressing medication non-adherence in patients with diabetes: a focus group study in a primary outpatient clinic
}

\author{
Elizabeth J Unni, ${ }^{1}$ Eve Van Wagoner, ${ }^{2}$ Olayinka O Shiyanbola ${ }^{3}$
}

To cite: Unni EJ, Van Wagoner E, Shiyanbola 00. Utilizing a $3 S$ (strategies, source and setting) approach to understand the patient's preferences when addressing medication non-adherence in patients with diabetes: a focus group study in a primary outpatient clinic. BMJ Open 2019;9:e024789. doi:10.1136/ bmjopen-2018-024789

- Prepublication history for this paper is available online. To view these files, please visit the journal online (http://dx.doi. org/10.1136/bmjopen-2018024789).

Received 13 June 2018 Revised 20 November 2018 Accepted 3 December 2018

Check for updates

(c) Author(s) (or their employer(s)) 2019. Re-use permitted under CC BY-NC. No commercial re-use. See rights and permissions. Published by BMJ.

For numbered affiliations see end of article.

Correspondence to Dr Elizabeth J Unni; eunni@roseman.edu

\section{ABSTRACT}

Objective Non-adherence with diabetes medicines is a challenge. Approximately $54 \%$ of patients are nonadherent with their diabetes medicines. The objective of this study was to understand patients' preferences when addressing non-adherence based on the $3 \mathrm{~S}$ approachstrategies (what approaches can be used in addressing non-adherence), settings (when and where should the intervention happen) and sources (who should provide the intervention).

Design A focus group research design was used.

Setting A primary outpatient clinic from an integrated healthcare system in the USA.

Participants Patients who were non-adherent with diabetes medicines.

Data collection The focus group guide was based on the Medication Adherence Reasons Scale, which has 19 reasons identified for non-adherence. For each item in the scale, patients were asked for strategies that can be used in addressing that issue, the setting in which the interventions should be provided and the source to provide the interventions.

Data analysis Open coding and content analysis. Results Two focus groups, each group having seven patients, were conducted. The major strategies identified were patient education, self-responsibility of patients, family support, reminders and societal support. The key educational needs were dealing with side effects and learning to use insulin properly, and a need for different learning styles when offering education. For the source, the major ones were physicians and pharmacists, having a continuous dialogue about the disease and medicines, and individuality in managing the disease. Respondents also recommended using a peer support group. For the setting, the patients preferred continuity of patient education throughout the disease.

Conclusion The 3S approach was able to elicit several recommendations from patients to improve their adherence with diabetes medicines. Educational strategies were identified as the foremost approach coming from physicians and pharmacists throughout their disease journey, in addition to peer support.
Strengths and limitations of this study

- The study used a focus group study design to identify solutions from the patient's perspective for each of the commonly found reasons of non-adherence.

- The study used a 3S (strategies, source and setting) approach by asking patients who were non-adherent with their medicines for intervention strategies, sources for intervention and settings for intervention.

- The focus group guide for the study was developed based on the comprehensive Medication Adherence Reasons Scale.

- The weakness is that the study was limited to patients covered by a commercial insurance from a university-based outpatient clinic.

- Patients with different demographics may have different solutions.

\section{INTRODUCTION}

Medication non-adherence is considered a silent epidemic. According to the WHO, approximately $50 \%$ of patients on medications for chronic conditions are non-adherent. ${ }^{1}$ Non-adherence costs the US healthcare system an estimated \$289 billion every year. ${ }^{2}$ It has been stated by the WHO that 'increasing the effectiveness of adherence interventions may have far greater impact on the health of the population than any improvement in specific medical treatments'. However, a major challenge faced in medication adherence research is the low success rate of interventions. Studies have shown that success rates with current interventions to improve medication adherence are as low as $4 \%-11 \%$, primarily because interventions are often developed based on literature reviews, clinician inputs and theoretical models. ${ }^{3}$ A potential deficit in this 
process of developing interventions may be the lack of patient input. This is concerning since the patient is the ultimate user of these intervention strategies.

Studies have shown that most providers use an informational style versus shared decision making when planning care for their patients. ${ }^{4}$ Improved communication that builds trust and rapport between the patient and the healthcare provider is often cited as one of the best ways to improve medication adherence. ${ }^{5}$ The required elements of patient-centredness in improving medication adherence are communication, shared decision making and support for self-management. ${ }^{5}$ Studies show that patients with chronic illnesses prefer a patient-centred approach because it meets their needs, preferences and values, and includes them in shared decision making. ${ }^{67}$

Shared decision making enables the application of patient-centred solutions to medication adherence and medication management problems based on the patient's perspective and their attitudes towards their treatment. However, in everyday clinical practice, with the limited time available for patient consultation, the patient may not have sufficient time to communicate with the provider regarding their needs and concerns about medication adherence. Additionally, the provider may not be able to come up with the most effective intervention strategy in the few minutes he or she has with the patient. If we can understand from the patient's point of view the intervention strategies that could work for them to overcome the various barriers to medication adherence, patient-centred interventions may be developed a priori and then implemented by providers. Likewise, patients could inform researchers regarding the potential source for an adherence intervention, that is, whether the intervention should be administered by someone specific within the healthcare team, such as the physician, the nurse, the pharmacist or someone else. Furthermore, patients are able to provide information on the best setting or timeline to deliver the adherence intervention (right after the diagnosis by the physician, during the follow-up call by the nurse or during a visit to the pharmacist). Thus, knowing the needs, preferences and values of patients can assist in developing patient-centred intervention strategies.

Non-adherence with diabetes medicines is especially detrimental. The reported non-adherence rate with diabetes medications is $53.6 \%$, and non-adherent patients have an annual inpatient cost $41 \%$ higher than the adherent patients. ${ }^{8}{ }^{9}$ In a disease where non-adherence can lead to significant future impairment (eg, dialysis, blindness, neuropathy and so on), it is essential for providers to understand why patients are non-adherent and how providers can impact patients' adherence with their diabetes medicines.

To develop patient-centred intervention strategies, it is essential to know what type of interventions are needed, who should provide it and when and how it should be provided. Thus, a 3S approach-Strategies, Source and Setting - was developed for this study's purpose. The aim of this study was to identify patient-proposed solutions to improve medication adherence in patients with diabetes using this $3 \mathrm{~S}$ approach. The specific objectives of this study were to understand what patients with diabetes perceive is needed for the development of a successful adherence intervention by gathering and defining the following information: (1) what intervention 'strategies' are recommended depending on the reason for non-adherence, (2) the preferred 'source' (physicians vs pharmacists vs nurse) to facilitate the intervention, and (3) the 'setting' to provide the intervention, such as the point of time in the disease (at the time of diagnosis, 3 months after diagnosis and so on) or the format for the delivery of the intervention (face to face or telephone, and so on).

\section{METHODOLOGY}

\section{Study design and sample}

The study used a qualitative focus group research design. The research team included two researchers with expertise in medication adherence and qualitative research, and an ambulatory care pharmacist. The researchers did not have any relationship with the patients.

Patients over the age of 18 with diabetes who were non-adherent with their diabetes medications were recruited for the study (EVW). The patients were recruited from a University of Utah outpatient clinic using convenience sampling. The inclusion criteria for patient eligibility were (1) diagnosed with type 2 diabetes (based on the International Classification of Diseases, 10th Revision codes), (2) prescribed diabetes medicines (from pharmacy claims database), and (3) members of the University of Utah health insurance who were non-adherent to their diabetes medicines based on the adherence measure, proportion of days covered (PDC). The study only included members of the University of Utah health insurance to ensure access to the patient's pharmacy claims database, so that the research team was able to track the medication adherence of these patients by calculating the PDC. Only patients who were non-adherent to their diabetes medicines were included in the study. The definition of non-adherence in this study was having a PDC less than $80 \%$. The Pharmacy Quality Alliance recommends $80 \%$ as the threshold for adherence with most chronic therapies. ${ }^{10}$ Any patient with critical illnesses such as cancer, in the last stages of life (with less than a year to live), with severe comorbidity (more than six chronic disease conditions), diagnosed with depression and non-English-speaking were excluded from the study. For these individuals, their reasons for medication non-adherence and coping skills may be different. The pharmacist manually searched the electronic health record to identify eligible patients. Once the patients were identified, the pharmacist invited these patients to participate in the study by telephone. If they agreed to be part of the study, they were called again and reminded about their study participation the day before the focus groups.

\section{Data collection}

The Medication Adherence Reasons Scale (MAR-Scale) was used as the reference in developing the focus group 
Box 1 Items from the Medication Adherence Reasons Scale that were used to develop the focus group guide

- I had side effects from this medicine.

- I did not have money to pay for this medicine.

- I was not comfortable taking it for personal reasons (eg, tired of taking medicines, too sick, my religious beliefs).

- I was not comfortable taking it for social reasons (eg, I was with friends).

- I don't think that I need this medicine anymore.

- I don't think that this medicine is working for me.

- I sometimes skip this medicine to see if it is still needed.

- I am concerned about possible side-effects from this medicine.

- I am concerned about long-term effects from this medicine.

- I had difficulty opening the container OR I had difficulty getting the injection ready to use (eg, opening the package, mixing contents, drawing out the medicine).

- I had difficulty swallowing this medicine OR I had difficulty with injecting this medicine (eg, fear of needles, physical or sensory problems).

- I didn't have the medicine because the pharmacy/provider was out of this medicine, I was out of refills, or the mail order did not reach in time.

- I didn't have the medicine because I didn't have a way to get to the pharmacy/provider.

- I am not sure how to take this medicine.

- I have trouble managing all the medicines I have to take.

- I would have taken it but simply missed it.

- I would have taken it but missed it because of busy schedule/ change in routine.

- I would have taken it but have difficulty remembering things in my daily life.

- I do not consider taking this medicine as a high priority in my daily routine.

guide (box 1). ${ }^{11}$ The MAR-Scale is a self-reported adherence measure with 19 reasons identified for medication non-adherence. It addresses most of the commonly reported reasons for non-adherence such as 'simple forgetfulness', 'skipping medicines', 'taking this medicine is not a priority' and so on. The MAR-Scale was developed from literature reviews and semistructured interviews with patients. ${ }^{11}{ }^{12}$ The scale has four major domains, namely non-adherence due to logistics, non-adherence due to beliefs, non-adherence due to long-term concern about medicines and non-adherence due to forgetfulness. ${ }^{11}$ The aim of the study was to use the $3 \mathrm{~S}$ approach to get feedback on the strategies, sources and settings for each of the four domains, so patient-centred interventions can be developed. For each item on the MAR-Scale, the patients were asked: 'What do you think should be done/communicated so that you will be adherent to your medicines?'

The 3S strategy was implemented to gather indepth feedback from patients on each item and domain. The patients were asked to express their opinion regarding potential intervention strategies. Patients' needs were assessed in terms of what can be done, said or offered by the providers. Patients were encouraged to reflect on their personal experiences or on what they have heard from their friends or families regarding reasons for non-adherence and then encouraged to give interventions that they believe would be best. Patients were also asked regarding their opinion on source, from whom they prefer to receive that information (physicians, nurses or pharmacists). Patients were then asked for their views on setting to better understand how often they want the information to be delivered to them and the best time to deliver that information. Finally, patients were asked to give their opinion on the best medium for communication (face-to-face communication, leaflets or telephone calls).

The primary investigator (EJU), who is an expert on medication adherence and qualitative research, moderated the focus group. As the developer of the MAR-Scale, the primary investigator is well versed with the various reasons for medication non-adherence. The primary investigator is an academician and does not have any established relationships with the patients. The study was explained to the patients while recruiting and before the focus groups. The focus group interviews took place in the conference rooms of the clinic and lasted approximately $90 \mathrm{~min}$. All the interviews were audio-recorded and were transcribed verbatim by the primary investigator. The participants were given a $\$ 25$ grocery store gift card and dinner for their participation.

\section{Data analysis}

Data were analysed using open coding and content analysis. The domains and items in the MAR-Scale as well as the $3 \mathrm{~S}$ approach served as a starting point for the open coding. The analysis started with repetitive reading of transcripts for immersion of data. The three study investigators worked independently to achieve data immersion, create initial codes, make notes and add additional notes with repeated reading, resulting in either editing the older codes or creating newer codes. Once the open coding was completed for each item in the MAR-Scale, these codes were further analysed to understand how they fit with the various MAR-Scale domains and the 3S approach. The investigators met and discussed their independent findings and any discrepancies. The discrepancies were discussed in further detail and the original transcripts were referenced as necessary, until agreement was reached. Once the themes were formed, categories were developed for strategies, sources and setting. All the analyses were conducted manually.

\section{Patient and public involvement}

There was no patient or public involvement in the development of research questions, design and data collection process in this study.

\section{RESULTS}

There were two focus groups with seven patients in each group. Of the 47 patients who were identified, 31 agreed to be a part of the study and only 14 actually participated. 
The mean age of the participants was 53.75 years (range: 32-78). On average, participants had been taking oral diabetes medicines for 10 years, ranging from 6 months to 30 years, and $41 \%$ of the participants were on insulin for an average of 4 years, ranging from 3 months to 9 years. The participants were taking an average of 5.66 medicines every day, ranging from 2 to 12 . Table 1 describes the major categories of patient-identified strategies, sources and setting to be better adherent with their medicines for diabetes.

\section{Strategies from the $3 S$ approach}

The patients identified seven major categories for adherence interventions (table 1). These included education, physician's role, pharmacist's role, patient's role, society, family support and reminders.

A major intervention strategy that emerged was patient education. The patients responded with very specific educational needs, such as a timeline on stopping medicines when they have side effects, probabilities of the side effects and proper use of medicines, especially insulin. The patients wanted both physicians and pharmacists to educate them, as well as pharmacists to perform follow-up calls. The patients also recommended the use of different learning styles when offering education.

The patients recognised the role of physicians in improving medication adherence. For example, they wanted physicians to maintain patient individuality while prescribing and managing diabetes, and to be mindful of the costs of the medicines. In addition to explaining the need and the long-term effects from the medicines to the patients, they also wanted physicians to have regular discussion regarding medicines and re-evaluate the necessity of medicines periodically so they remained assured of the benefit.

The patients identified the role of pharmacists for cost control, continuous access to medicines and education. To reduce costs and improve access, the patients suggested various strategies such as automatic 90-day refills, use of coupons, home delivery of medicines, offering pillboxes and identifying social services to assist with medication costs. Additionally, the patients wanted pharmacists to focus on their medication education. Patient education needs included teaching them how to use their medicines, especially insulin injections, and insurance coverage especially for supplemental services such as transportation.

The study participants advocated for a patient-centred role in improving medication adherence. They strongly recommended the need for patients to communicate with physicians before stopping medicines. Another strategy recommended by the patients were diabetes support groups since their peers understand their needs and concerns better and provide support and encouragement. The patients perceived that this would allow them to come together monthly or bimonthly to discuss their issues, disease management strategies, recipes for diet management and so on.
The patients also recognised a societal role for improving medication adherence. A major intervention strategy voiced by the participants was the need for private spaces for injecting diabetes medication when they were in public. Privacy was more an issue with young patients and non-whites, who felt more uncomfortable injecting in public. The respondents also mentioned the need to increase awareness about diabetes and diabetes management to increase the social acceptability of the disease.

Family plays an important support role in improving medication adherence. Support from families included opening pill bottles for them, and reminding and encouraging them to take their medicines. The patients also wanted families to be more sensitive to the difficulties faced by them, such as diet restrictions, and suggested educating families on diabetes and diabetes management so that they are more empathetic.

Since forgetfulness was one of the most commonly reported reasons for non-adherence, reminders was one of the strategies recommended by the participants. The various reminder interventions recommended by the participants included pillboxes, visual aids, organising medicines, alarms and making a conscious effort to remember to take the medicines when there is a change in schedule.

\section{Sources for the intervention}

Four major sources for interventions became evident from the focus groups. These included physician's office, diabetes educator, support coordinator and the healthcare team. From the physician's office, the patients wanted education as to why they are taking the medicine and reinforcement about the absolute necessity of taking medicines as prescribed. The patients wanted to be part of the decision-making process as well. From the diabetes educator, they wanted a more indepth education about the disease and disease management. Another interesting source they wanted was a support coordinator who schedules and facilitates support groups. The patients also understood the importance of having a healthcare team for diabetes management, especially since diabetes is a 'lifelong disease journey'.

\section{Setting for the intervention}

The patients reiterated the importance of continuity of information as the disease and the disease management change over time, and regular feedback was preferred to keep up with the changes. There was no preference for the method of communication, such as face to face or telephone. The emphasis was on communication and the continuity of information. The patients echoed the ambiguity in diabetes management especially in the initial years and the need for constant communication from the providers. Although they agreed that information given to them right at the point of diagnosis might not be that useful, they still wanted some information. Additionally, changes such as medication dosage happen throughout the disease, warranting continuity of information. The 
Table 1 Categories of intervention strategies, sources and setting

Category Theme Sample quote

Strategies

Education

When to stop taking a medication due to

"putting a time line on it will help you a little better..." side effects (timeline).

Probabilities for the side effects (statistics).

"...say well one out of a hundred thousand usually get this side effect and it usually lasts for 2 months..."

Pharmacies educating patients how to use the insulin.

"When I first started it, the pharmacist just told me how to do it. I'm one of those type of people that has to do it in order to learn it and just put it in...pull it out..."

Pharmacists doing follow-up calls on using insulin.

"Then the pharmacist downstairs keeps tabs on me and you know so they'd call me every other week to see how I'm doing and uh to see how my blood is..."

Pharmacists instructing the patients when there is a change in the dosage.

"No, it's not that silly. Seriously, I did not know it was 4 pills... Cause it's always been one time a day until they changed the size of the pills. Then it went from one time a day to two times a day and they broke the pills into halves so that's 4 pills instead of just 2 pills..."

Incorporating different learning styles.

Physicians $\quad \begin{aligned} & \text { Need individuality to prescribing and } \\ & \text { management of diabetes. }\end{aligned}$

"...but not everybody learns the same."

"So, I feel a lot of times that those that have either lifelong diseases or ones that are probably just going have this forever such as diabetes that everybody is sort of liked up to one big this is what we are going to do for everybody and there's no individuality to it..."

Have to explain the need of medicines. "...he said you're diabetic and gave me metformin that was it...and when I came back later it was 249 he said that's the right range and so he never really explained what was going on and where I needed to be or anything like that..."

Need to explain the long-term effects (need) of the medicines.

"Well with diabetes I wish they would tell you more it's a long term effects...creep up on you one day you can feel fine the next day or when I woke up the next day I could not see my hand before my face..."

Re-evaluate the necessity of medications “...but I think just through the whole journey like I'm on periodically so that the patients are reassured. something I always go to the doctor and say Do I really need to take this? I don't know that it's doing any good and I'd rather not take it. But you know I want to take as little as I can..."

Having regular talks with the patients about the medicine.

"...So, at least I appreciate the fact that she is open to discuss it with me every time I go in and I bring that up again and she at least is very open to discuss that with me and um I appreciate that I think that's important to kind of put your mind at ease okay I am concerned about these long term effects but my doctor is taking the time we're talking about it and we'll go another 3 months..."

Showing patients how to use the insulin. "My doctor had me go downstairs and get my medicine and come back up and they showed me how to use my insulin..."

Prescribing drugs that are covered by insurance.

"I went to my physician before I came here and I told her that my insurance changed like... So my doctor took the time she actually went in and oh, your insurance will cover this She switched all my medicines and stuff..."

Continued 
Table 1 Continued

\begin{tabular}{lll}
\hline Category & Theme & Sample quote \\
\hline Pharmacists & $\begin{array}{l}\text { Logistics regarding opening pill bottle- } \\
\text { asking if the patient want non-child- }\end{array}$ & "I think they should ask you if you want child proof..." \\
& resistant containers and avoid dispensing bottle and just put your label on there... Those are the worst
\end{tabular}
in manufacturer's bottles. try to open..."

Educating patients how to inject the medicine-device training/refer patients to classes for device training.

Should offer alternatives if patients cannot swallow.

"If there was an alert in their system: Hey, you're starting this new medication. Do you want us to show you how to use it?"

Working with patient to ensure accessautomatic 90-day refills, delivering medicines to home.

"But they did give me alternatives. Liquid metformin which is horrible. I didn't take it because it was so horrible."

"I don't think we should have to ask for a 90 day. It should just automatically..."

Working with patient to reduce costautomatic 90-day refills, coupons, identifying social services for patients who cannot afford medicines.

"Yeah, but they didn't offer me a discount...It would have helped if they were like well, let's see if we have a coupon.

That's really nice..."

"I was just gonna say there's a lot of social services out there that can help also. I don't know if the pharmacy has any kind of case manager attached to it or doctor's office did, but you can contact them yourself and social services, LDS social services, they'll know through groups that sponsor people that can't afford their medications..."

Technicians helping with understanding copays.

"...we have specialized pharmacy technicians who start looking at your various insurance have books of what's covered and what isn't what copay there are..."

Educating patients about insurance coverage for transportation services.

"My insurance company calls me every so often because of one of the medications I am on. I am not supposed to stop taking it at all and I did stop taking it and they were right on top of me on that but...uhm...she asked me why and I explained I had the kids and I can't get out to the pharmacy and get back home all within a reasonable amount of time and she goes, we have transportation and l'm like sign me up."

Offering pillbox for medicine management.

"My pill box came from my pharmacist that calls me every month to make sure I am taking my medication."

Patients Responsibility of taking medicines as

"Okay I have one doctor how many patients does that doctor committed. have? So isn't it my responsibility for my own health not his he's not God he's not going to fix things he's helping me fix myself..."

Need to talk to the physicians before stopping the medicines.

"I didn't just stop taking them. I went in and talked to him and he told..."

Form diabetes support groups to help each other.

"Because I did not know what I could eat or what I couldn't eat I stopped eating altogether I was just drinking water because I was so scared that I would eat something that would kill me..."

Society

Have a private area for injecting in public. "Like me and him were in the restaurant. Just stopped and made him like turn so no one saw what I was doing I hope they don't look over hey, what are you doing? You have a place for moms to breast feed, right? Do you have a diabetic room?"

Making it more socially acceptable with "Think about those little guys. juvenile diabetes they have to take that medicine They don't have a choice. either just like my niece other kids are seeing them do that and it's not normal and if there were a way to put a billboard up to show it is normal not just for juvenile diabetes, but diabetes in general that people do have to carry needles and Insulin I think that would be a very good idea..." 
Table 1 Continued

\begin{tabular}{|c|c|c|}
\hline Category & Theme & Sample quote \\
\hline \multirow[t]{2}{*}{ Families } & $\begin{array}{l}\text { Supporting patients in taking medicines } \\
\text { as needed/reminding them/opening } \\
\text { containers/managing them. }\end{array}$ & $\begin{array}{l}\text { "I have a little } 5 \text { year old that says, 'Mimi...Don't forget those } \\
\text { pills you know...they keep you alive for me..." "' } \\
\text { "Well my husband is the voice of reason. He's in the medical } \\
\text { field and so he explains to me why you should, if you don't do } \\
\text { it, this could happen..." }\end{array}$ \\
\hline & Being more sensitive. & $\begin{array}{l}\text { "... when I'm with my family drinking and eat that's what we do } \\
\text { when we get together to drink and we eat so I'll get made fun } \\
\text { of because I'm diabetic it starts to affect me..." }\end{array}$ \\
\hline
\end{tabular}

Reminders Pillboxes.

“...taking right now. 10, 11, 12 medicines. No it's more than that but, what l've done is that I've taken one of those 30 day pill box..."

Visual aids.

"... On my fridge I have a picture of my insulin just so when I pass it I can say, oh insulin."

Organising medicines.

"See, I try to make sure I take them at the same time, everyday...And every night and I keep them in order so I take metformin then my naproxen then my um whatever is next..."

Alarms.

"...But I try really hard to remember to take them. I even got an alarm set. And now I take them every day at the same time. So that they work properly."

Making effort to remember the medicines "I think there's more adjustment to be made when you take when there is change in schedule. insulin injection."

\section{Source}

Physician's office.

"I want to know why you want me to do this and she took the time to do that. And I think a doctor should If I'm gonna see you and I'm gonna pay you to be my doctor you're gonna spend some time and answer my questions so I can help make the decision rather than you just deciding for me."

Diabetes educator.

"They break it down like this is... They gave me this map thing. You should have like this much green stuff like that to help you so you can know oh this is what my diet should look like..."

Support coordinator.

"Yes. Somebody that can get us all together and say this is the day we are meeting. This is the time we are meeting. Come on in. Let's all just sit down and have a discussion."

Healthcare team.

"I think it is a little bit of all of them... That's what I was going to say because if you're diagnosed with something most likely going to be with you your whole life or it is going to be with you your whole life and I think there should be a team of people that can kind of walk you through it all. Know what I mean?"

Needs continuity in information throughout the disease.

Needs information both right at diagnosis and later on.

Educating patients on proper injecting techniques for at least the first 6-12 months after diagnosis.
“My first 2 or 3 years of being diabetic. Ambiguity..."

“When you're first diagnosed, I don't know about you guys, but I was in shock. I couldn't hear. I mean I had just had a freaking heart attack. What are you telling me I have diabetes for?"

"Yeah, like I said, I didn't know to switch sites to rotate...I don't want to do it hurts..." patients also reinforced the necessity to educate patients with proper insulin injection techniques for at least 6-12 months after diagnosis.

\section{DISCUSSION}

The goal of the study was to understand patient-proposed suggestions and recommendations in developing 
interventions to improve medication adherence in patients with type 2 diabetes. The recommendations were derived from a 3S approach-strategy, source and setting. The patients identified education as the major strategy to improve medication adherence, and they were specific about their expectations from each healthcare provider. Additionally, the patients realised the significance of social support and the continuity of information provided to them as diabetes is a lifelong journey. Although there is vast literature on intervention to improve medication adherence among patients with type 2 diabetes, there is limited literature on incorporating the preferences of patients into the development of these interventions. A recent study by McMullen $\mathrm{et} \mathrm{al}^{13}$ engaged various stakeholders such as patients, providers, researchers, payers, policy makers, product makers and funders in identifying and prioritising adherence interventions and medication management. ${ }^{13}$ Compared with the existing literature, the strengths of this study are that it focused on one disease condition and used a focus group study design to identify the solutions from the patient's perspective for each of the commonly found reasons of non-adherence.

As can be seen from the results, education was a major strategy recommended by study participants in improving adherence to diabetes medications. This is similar to the results from McMullen et al. ${ }^{13}$ However, the results from this study also demonstrate the specific educational needs of the patients. Interestingly, the patients wanted the crucial part of education, the necessity of medicines, coming from the physicians themselves. Zullig et al, in describing successful and scalable interventions, point out that, although educational interventions are important, it can come from other health professionals such as health educators or nurses. ${ }^{14}$ However, the proposed strategy from the patients in this study underscores findings from prior literature that patients' beliefs about their medicines and illnesses are important factors that contribute towards medication adherence. Once patients are convinced of the necessity of taking medicines and that the medicines are individualised for them by their physicians, they are open to further education from other sources such as diabetes educators. The major education they needed from pharmacists were techniques in taking their medicines correctly, such as proper injection techniques. All these educational strategies show how patients look at various members of the healthcare team for different educational needs.

Ensuring continuous and economical access to medicines was important for patients as they believed that pharmacists were the most prepared to carry out this aspect of an adherence intervention with their knowledge about insurance coverage. While being mindful about the fact that the society and families may lack understanding about diabetes and diabetes management, the patients wanted the society and family to be empathetic and more accepting of patients with diabetes. These results are similar to the results from the literature that has shown a positive correlation between family/social support and diabetes management and improved adherence. ${ }^{15} 16$

A significant source for the delivery of adherence interventions that were reported by the study participants was the use of a support group. McMullen $e t a l$ s study results also reported that peer support was a priority for patients, although not for researchers. ${ }^{13}$ The participants felt that having a monthly or bimonthly meeting on diabetes management among patients could be more hands-on and beneficial in addition to meeting with the providers. The participants reported that support groups could be used for sharing successful strategies for improved medication adherence and better management of the disease. The impact of using peer support on chronic disease management is established in the literature, and the success from peer support is attributed to gaining mastery of tasks and improving clinical outcomes based on the sharing of experiences. ${ }^{17-19}$

Another major observation from this study was how patients prioritised the continuity of information needed for improved medication adherence. Realising that the management of diabetes is a lifelong journey, participants wanted the providers, especially the physician, to have a continuous dialogue with them on the disease as well as the management of the disease. For them, this dialogue should start from the day of disease diagnosis and should be continued over time. Probably, these consistent conversations would empower patients to be better decision makers regarding disease management. The continuity of information corresponds with the literature that provides adequate evidence on the importance of physician-patient communication in disease management. ${ }^{20-24}$

The study has some limitations. The study only had two focus groups. However, the analysis of the data showed data saturation. The fact that the data were analysed by three investigators with different professional and educational backgrounds and were able to reach consensus demonstrates data adequacy. While the primary investigator was an expert on medication adherence and the developer of the MAR-Scale, which was used as the basis for the focus group guide, the second author was a clinical pharmacist and the third author an expert on qualitative methodology. All the study participants were from the same clinic which is part of a university hospital, which can limit the generalisability of the study. One exclusion criterion for the study was that patients with more than six comorbid conditions should be excluded. Although multimorbidity is becoming more common, we excluded those patients since their reasons for non-adherence and coping skills can be different from other patients. However, this exclusion can decrease the representativeness of the sample.

Further studies are needed to determine if patients from different settings (such as Medicaid, Medicare and commercially insured population not in a university setting) have the same recommendations to improve medication adherence. Clinical studies incorporating interventions based on the findings from the study 
are also needed to determine the effectiveness of the patient-proposed interventions.

\section{CONCLUSION}

A complex problem such as medication non-adherence needs patient-centred intervention for its success. The $3 \mathrm{~S}$ approach used in eliciting input from the patients about medication adherence strategies, source and setting provided valuable information that can be used in developing adherence interventions. The patients identified education as the major strategy and their expectations from each healthcare provider. Additionally, the patients realised the significance of social support and the continuity of information provided to them as diabetes is a lifelong journey.

\section{Author affiliations}

${ }^{1}$ Department of Pharmaceutical Sciences, College of Pharmacy, Roseman University of Health Sciences, South Jordan, Utah, USA

${ }^{2}$ Pharmacy Primary Care Services, University of Utah Health, South Jordan, Utah, USA

${ }^{3}$ Division of Social and Administrative Sciences, University of Wisconsin-Madison, Madison, Wisconsin, USA

Contributors All the three authors met all the four author criteria established by the ICMJE. EJU: study design, data collection, data analysis and reporting. EVW: patient recruitment, data analysis and reporting. 00S: data analysis and reporting.

Funding The authors have not declared a specific grant for this research from any funding agency in the public, commercial or not-for-profit sectors.

Competing interests None declared.

Patient consent Not required.

Ethics approval The University of Utah Institutional Review Board approved the study as exempt.

Provenance and peer review Not commissioned; externally peer reviewed.

Data sharing statement Due to the qualitative nature of this study, the data are written transcripts of patient focus groups. Although participant information is deidentified, there are references to their experiences with taking medicines; thus, we prefer to not make these transcripts publicly available.

Open access This is an open access article distributed in accordance with the Creative Commons Attribution Non Commercial (CC BY-NC 4.0) license, which permits others to distribute, remix, adapt, build upon this work non-commercially, and license their derivative works on different terms, provided the original work is properly cited, appropriate credit is given, any changes made indicated, and the use is non-commercial. See: http://creativecommons.org/licenses/by-nc/4.0/.

\section{REFERENCES}

1. Burkhart PV, Sabaté E. Adherence to long-term therapies: evidence for action. J Nurs Scholarsh 2003;35:207.
2. New England Health Care Institute. Thinking outside the pillbox: a system-wide approach to improving patient medication adherence for chronic disease. 2009. Acessed at https://www.nehi.net/writable/ publication_files/file/pa_issue_brief_final.pdf (Accessed 12 Jun 2018).

3. Peterson AM, Takiya L, Finley R. Meta-analysis of trials of interventions to improve medication adherence. Am J Health Syst Pharm 2003;60:657-65.

4. Lawson MT. Nurse practitioner and physician communication styles. Appl Nurs Res 2002;15:60-6.

5. Zolnierek KB, Dimatteo MR. Physician communication and patient adherence to treatment: a meta-analysis. Med Care 2009;47:826-34.

6. Frosch DL, Moulton BW, Wexler RM, et al. Shared decision making in the United States: policy and implementation activity on multiple fronts. Z Evid Fortbild Qual Gesundhwes 2011;105:305-12.

7. Makoul G, Clayman ML. An integrative model of shared decision making in medical encounters. Patient Educ Couns 2006;60:301-12.

8. Feldman BS, Cohen-Stavi CJ, Leibowitz M, et al. Defining the role of medication adherence in poor glycemic control among a general adult population with diabetes. PLoS One 2014;9:e108145.

9. luga AO, McGuire MJ. Adherence and health care costs. Risk Manag Healthc Policy 2014;7:35-44.

10. PQA adherence measures. Accessed at https://www.pqaalliance.org/ adherence-measures (14 Sep 2018).

11. Unni EJ, Olson JL, Farris KB. Revision and validation of Medication Adherence Reasons Scale (MAR-Scale). Curr Med Res Opin 2014;30:211-21.

12. Unni EJ, Farris KB. Development of a new scale to measure self-reported medication nonadherence. Res Social Adm Pharm 2015;11:e133-43.

13. McMullen CK, Safford MM, Bosworth HB, et al. Patient-centered priorities for improving medication management and adherence. Patient Educ Couns 2015;98:102-10.

14. Zullig LL, Gellad WF, Moaddeb J, et al. Improving diabetes medication adherence: successful, scalable interventions. Patient Prefer Adherence 2015;9:139-49.

15. Mayberry LS, Osborn CY. Family support, medication adherence, and glycemic control among adults with type 2 diabetes. Diabetes Care 2012;35:1239-45.

16. Miller TA, Dimatteo MR. Importance of family/social support and impact on adherence to diabetic therapy. Diabetes Metab Syndr Obes 2013;6:421-6.

17. Wilson W, Pratt C. The impact of diabetes education and peer support upon weight and glycemic control of elderly persons with noninsulin dependent diabetes mellitus (NIDDM). Am J Public Health 1987;77:634-5.

18. Gilden JL, Hendryx MS, Clar S, et al. Diabetes support groups improve health care of older diabetic patients. J Am Geriatr Soc 1992;40:147-50.

19. Lorig KR, Sobel DS, Ritter PL, et al. Effect of a self-management program on patients with chronic disease. Eff Clin Pract $2001 ; 4: 256-62$.

20. Von Korff M, Gruman J, Schaefer J, et al. Collaborative management of chronic illness. Ann Intern Med 1997;127:1097-102.

21. Ciechanowski PS, Katon WJ, Russo JE, et al. The patient-provider relationship: attachment theory and adherence to treatment in diabetes. Am J Psychiatry 2001;158:29-35.

22. Jahng KH, Martin LR, Golin CE, et al. Preferences for medical collaboration: patient-physician congruence and patient outcomes. Patient Educ Couns 2005;57:308-14.

23. Street RL, Krupat E, Bell RA, et al. Beliefs about control in the physician-patient relationship: effect on communication in medical encounters. J Gen Intern Med 2003;18:609-16.

24. Heisler M, Bouknight RR, Hayward RA, et al. The relative importance of physician communication, participatory decision making, and patient understanding in diabetes self-management. $J$ Gen Intern Med 2002;17:243-52. 